\title{
Effects of Relaxation Exercise and Sleep Environment Modification on Stress among Institutionalized Elderly in Nairobi City, Kenya
}

\author{
Githang'a J.W, Gitahi, T, Mugala, H. Bulinda* \\ Lecturer, Kenyatta University, Department of Physical Education Exercise and Science, Kenya
}

*Corresponding Author: H. Bulinda, Lecturer, Kenyatta University, Department of Physical Education Exercise and Science, Kenya

\begin{abstract}
Stress is a key factor in reducing the efficacy of the treatment of high blood pressure and poor sleep quality in the elderly. High blood pressure and poor sleep quality are important preventable contributors to disease and death. Inadequate sleep is one of the most common side effects of stress resulting in reduced sleep quality. To effectively manage stress, the ability to relax and create a sleep inducing environment is paramount. Due to the negative impact of stress on human health, many types of stress management therapies have been put forward for the elderly in order to decrease stress and promote wellbeing. The purpose of this study was to assess the effectiveness of Progressive Muscle Relaxation (PMR) exercise and sleep environment modification on stress level of residents aged 60 years and above in a home for the aged in Nairobi County, Kenya. The study aimed at highlighting the benefits of PMR together with the modification of sleep environment by use of sleep masks in management of stress of both male and female elderly citizens. The study adopted an experimental pre-test post-test control group design.A total of 46 participants were randomly selected from the home to create experimental and control groups with 23 participants in each group. After ethical clearance and research permit approval, data was collected using Perceived Stress Scale (PSS). The psychological variable was assessed on both experimental and control groups at pre-test and post-test. A total of 43 (93.5\%) participants successfully completed the two months intervention programme. In the experimental group, PMR exercise was performed in 45 minutes sessions, three times weekly for two consecutive months and the participants used sleep masks during the entire intervention period. The control group, however, continued with their normal routine activities throughout the intervention period. For the purposes of comparison, data was collected in both experimental and control group. The data was coded and analyzed using Statistical Package for Social Sciences (SPSS) version 20. The results of repeated measures ANOVA and Post Hoc tests between experimental and control groups showed that there were statistically significant differences at $p \leq 0.05$ between the pre-test and post-test scores on stress level, $F=18.969, p=0.001$. It was concluded that PMR and use of sleep masks were effective intervention measures in the elderly with high stress level. The study recommended that PMR and sleep masks programmes should be expanded in the community areas with larger population of the elderly.
\end{abstract}

Keywords: Light at Night, Progressive Muscle Relaxation Exercise, Sleep Environment, Sleep Mask, Stress

\section{INTRODUCTION}

The elderly persons in Kenya are faced with stress emanating from a host of problems that vary from economic, health, social to personal problems (Olum, 2014). It is highlighted that $43 \%$ of adults experience hostile health effects from stress. This outcome contributes to 75-90\% of hospital visits associated with stress-related conditions and complaints including high blood pressure and poor sleep (Bruno, 2013; Harvard Health Publications [HHP], 2009; Olum, 2014). Relaxation techniques for the older persons include a number of practices such as progressive relaxation, autogenic training, guided imagery, biofeedback, self-hypnosis, and deep breathing exercises (Benson, Casey \& Dadoly, 2008; HHP, 2009; National Center for Complementary and Integrative Health [NCCIH], 2016). One of the most effective techniques is the Progressive Muscle Relaxation (PMR) exercise, which is not only simple to perform but also has better reported results oriented towards managing distress and poor sleep quality associated with stress in this population (Barnes, Powell-Griner, McFann \& Nahin, 2004; Muriungi \& Ndetei, 2013; Shinde et al., 2013).

The elderly are also susceptible to irregular night-time awakening due to the impaired levels in 
melatonin, a hormone that enhances sleep quality (Fulghum, 2007; Wilson, 2016), that is also associated with aging (Drake, Mills \& Noble, 2004). In addition, inappropriate sleep environment due to lighting in the room has been found to interfere with melatonin synthesis. Researchers recommend that the bedroom should be kept as dark as possible with the help of blackout drapes or sleep masks (Bonnet \& Arand, 2016; Healthwise Staff, 2015; The Alternative Daily, 2016). Fulghum (2007) asserts that melatonin as a factor in sleep restoration also extends its powerful antioxidant effect to the enhancement of the brain and nervous system. This helps to protect against age-related damage and consequently leading to a tremendously improved quality of life among this significant population (Figueiro, 2015; Obayashi, Saeki, Iwamoto, Ikada \& Kurumatani, 2013).

Stress and Light At Night (LAN), both inhibit adequate sleep in this population. Sleep loss is deemed to impair the elderly from living a healthy, safe and focused life and ironically the ability to enjoy good sleep may be under their control (Engle-Friedman, 2014). As a possible solution, the elderly are encouraged to access bright light in the morning and during the day preferably from the sun and avoid LAN in the evening by sleeping in a completely dark bedroom (Stevens, 2015). One of the pivotal goals of relaxation exercises and good sleep hygiene is to reduce anxiety over good sleep quality. Studies have shown that relaxation exercise and sleep hygiene are two healthy, nonpharmacological interventions that not only help the older adults fall asleep faster but also to awaken feelings of refreshed and relaxed state (AHRQ, 2005; Yang et al., 2012).

Sheu et al., (2003) conducted a study in Taiwan that examined the effect of PMR on blood pressure and psychosocial status in clients with essential hypertension. The training had an immediate effect, reducing pulse rate by 2.35 beats $/ \mathrm{min}$, systolic blood pressure by $5.44 \mathrm{mmHg}$, and diastolic blood pressure by $3.48 \mathrm{mmHg}$. After 4 weeks of PMR training, further decreases in pulse rate (2.9 beats/min), systolic blood pressure $(5.1 \mathrm{mmHg})$, and diastolic blood pressure $(3.1 \mathrm{mmHg})$ occurred. PMR significantly lowered patients' perception of stress, and it enhanced their perception of health. The study concluded that PMR is beneficial for patients with essential hypertension, and nurses may use it to enhance their independent function as well as their quality of life.

Schneider, Alexander, Staggers and Orme-Johnson (2005) tested the short-term efficacy and feasibility of Progressive Muscle Relaxations as one of the mental and physical stress reduction approaches in the treatment of mild hypertension in older African Americans. Progressive muscle relaxation lowered systolic pressure by $4.7 \mathrm{mmHg}$ and diastolic pressure by $3.3 \mathrm{mmHg}$.

Hamidizadeh et al., (2005) conducted a quasi-experimental research to evaluate efficacy and feasibility of PMR on blood pressure of elderly subject with mild and moderate hypertension residing at Kahrizak Charity Foundation for elderly. The study concluded that Progressive Muscle Relaxation (PMR) reduces the blood pressure in subjects practicing this program. In an analysis by Obayashi et al., (2013) on effect of Light At Night (LAN) on depression in the general elderly population, the investigators concluded that chronic exposure to bright light in the home setting was significantly associated with depression. The researchers further recommended that based on their findings, the affinity for depression in the elderly may be lowered by ensuring total darkness in the bedroom when sleeping. Kumutha et al., (2014) employed a pre-test and post-test control group design to assess the effects of PMR on stress levels and blood pressure in hypertensive elderly in India. The researchers concluded that PMR exercises were beneficial to the hypertensive elderly as the programme led to the reduction of stress and blood pressure.

\section{METHODOLOGY}

The objective of the study was to determine changes in the pre-test stress levels of the elderly following administration of treatment at post-test. Based on this, the study, an experimental by design hypothesized that there would be no significant differences in stress levels of the elderly in institutionalized homes in Nairobi, Kenya at pre-test, mid-test and post-test. The study ran for duration of three month in a home of the elderly in Nairobi County Kenya and involved a total of 46 respondents, half of these in the experimental and another half comprising of the control group.

The study observed inclusion criteria where respondents had to be over 60 years old, able to follow instructions in Kiswahili and / or English language, capable of giving informed consent and ability to wear sleep masks throughout the duration of sleep and could perform the muscular relaxation 
exercises as prompted. The study excluded those who were cognitively impaired or fail to carry out simple instructions on muscle relaxation exercise and use of sleep masks.

Subsequent to enrollment, the researcher arranged mutually agreeable date and time to meet the participants for screening and establishing pre-test assessment scores on stress level. The PSS questionnaire was administered to the experimental and control group in the form of an interview setup by the investigators and the research assistants. The participants were issued with sleep masks and instructed on how to use them at bedtime as blindfolds during the intervention period under the supervision of caregivers. All participants were evaluated within 48 hours before and after the intervention.

Data collection was carried out by the Progressive Muscle Relaxation (PMR) protocol sheet together with a stopwatch to implement the relaxation technique. This protocol has standardized and validated procedures of a classic muscle relaxation program by Jacobson citation. Data on self-report measures of stress index was collected using the Perceived Stress Scale (PSS). Sleep masks were used to create total darkness in the sleep environment.

Participants in the experimental group were assigned to an organized eight week group therapy of PMR programme engaged by the researcher and PMR instructors. The PMR intervention included a total of 24 sessions, held three times a week; each for duration of 40 minutes. This technique involved systematically relaxing the major muscle groups of the body with the goal of physical and mental relaxation. The PMR technique consisted of two processes namely tensing and relaxing of muscle groups, tensing for five seconds and relaxing for 10 seconds. The first session included an introductory group discussion about stress and the subjects were taught how to relax and contract 16 muscle groups. The subjects sat up, comfortably covered with a warm blanket, closed their eyes and assumed a serene demeanor as instructed in the PMR protocol. Classes for the programme were scheduled at the same time in the morning (0900 -1200 hours) and in similar settings, referred to as the therapy room.

The subjects in the control group were encouraged to follow their daily routine activities which included taking regular medication, regular blood pressure checking and dietary management and not to begin any new exercise program. The participants however were compensated and received same PMR and sleep environment modification interventions for two months after completion of the study.

The interventions were delivered by the same set of instructors who had experience in PMR training. To avoid data bias, care was taken to ensure that PMR and use of sleep masks for the experimental group was the only content of the group sessions for the PMR group. All the intervention research assistants were instructed not to personally influence either by encouraging or discouraging the participants beyond intended interventions. These therapeutic sessions were led by the researcher and research assistants for two months after which a post-test was administered using the same stress index questionnaire to the test and control group.

Statistical analysis was done by using Statistical Package for Social Sciences (SPSS) version 20 using descriptive statistics of means, standard deviation (SD) and repeated measures of ANOVA to tests significance. Post Hoc (Scheffe's Confidence Interval) tests were done to compare differences between pre, mid and post sessions of the treatment

\section{FINDINGS}

The mean differences in stress levels at pre-test, mid-test and post-test between experimental and control groups are shown in Table 1.

Table1: Mean Differences in Stress Level between Experimental and Control Group

\begin{tabular}{|l|l|l|l|}
\hline \multicolumn{1}{|c|}{ Testing Phase } & \multicolumn{1}{c|}{ N } & \multicolumn{1}{c|}{ MD (E-C) } \\
\cline { 1 - 3 } Pre-test & \multicolumn{1}{|c|}{3.20} \\
\hline Experimental & 22 & $24.32 \pm 5.73$ & \\
\hline Control & 21 & $21.12 \pm 5.72$ & \multirow{2}{*}{-3.78} \\
\hline Mid-test & $16.41 \pm 7.74$ & \\
\hline Experimental & 22 & $20.19 \pm 6.39$ & \\
\hline Control & 21 &
\end{tabular}


Effects of Relaxation Exercise and Sleep Environment Modification on Stress among Institutionalized Elderly in Nairobi City, Kenya

\begin{tabular}{|l|l|l|l|}
\hline Post-test & \multicolumn{2}{|l|}{-6.48} \\
\hline Experimental & 22 & $12 \pm 6.25$ & \\
\hline Control & 21 & $18.48 \pm 5.15$ & \\
\hline
\end{tabular}

The pooled means of stress level scores at pre-test for the experimental and control groups showed very high stress levels $(\bar{X}=24.32 \pm 5.73$ and $\bar{X}=21.12 \pm 5.72)$ respectively. At mid-test, the mean for both experimental and control group displayed high stress levels $(\bar{X}=16.41 \pm 7.74$ and $\bar{X}=20.19 \pm 6.39)$ respectively. There was a mean difference of -3.78 in the stress level between experimental and control groups at mid-test indicating a decline in stress level in the experimental group at mid-test.

The post-test results in Table 1 showed that the experimental group had a mean $(\overline{\mathrm{X}}=12 \pm 6.25)$ showing average stress levels while the control group recorded high stress levels $(\bar{X}=18.48 \pm 5.15)$. The table also indicates that there was a mean difference of -6.48 in the stress scores between experimental and control groups between the two test phases. These findings indicate that there was a decline in stress level in the experimental group.

To determine whether the differences in stress levels at pre-test, mid-test and post-test between the experimental and control groups were significant, repeated measures ANOVA and Post Hoc (Scheffe's Confidence Interval) tests were conducted. The results of the tests are in Table 2.

Table2: Analysis of Variance (ANOVA) on Stress Level at Pre-test, Mid-test and Post-test

\begin{tabular}{|l|l|l|l|l|c|}
\hline & \multicolumn{1}{|c|}{ Sum of Squares } & df & \multicolumn{1}{c|}{ Mean Square } & $\boldsymbol{F}$ & $\boldsymbol{p}$ \\
\hline Between Groups & 120.334 & 1 & 120.334 & 18.969 & $.001^{*}$ \\
\hline Within Groups & 1011.938 & 2 & 505.969 & & \\
\hline Total & 1132.272 & 3 & & & \\
\hline
\end{tabular}

*Significant at the $p \leq 0.05$ level

The repeated measures ANOVA results in Table 2 show that the mean difference in stress level between the experimental and control groups was statistically significant $(\mathrm{MD}=-3.78 ;-6.48, F=$ $18.969, \mathrm{df}=1, p=.001)$.

It was therefore concluded that there was significant difference between the experimental and control groups in the stress levels. To assess which testing phases were different from the other, a post-hoc (Scheffé's Confidence Interval) test was done to compare the means at mid-test and post-test. The results are shown in Table 3.

Table3: Post-Hoc Test on Phase of Testing and Stress Level

\begin{tabular}{|l|l|l|}
\hline \multicolumn{1}{|c|}{ (I) Experimental/Control } & \multicolumn{1}{|c|}{ (J) Experimental/Control } & \multicolumn{1}{c|}{ Mean Difference (I-J) } \\
\hline Pre-test & Mid-test & $3.788(*)$ \\
\hline & Post-test & $6.850(*)$ \\
\hline Mid-test & Pre-test & $-3.788(*)$ \\
\hline & Post-test & $3.062(*)$ \\
\hline Post-test & Pre-test & $-6.850(*)$ \\
\hline & Mid-test & $-3.062(*)$ \\
\hline
\end{tabular}

*The mean difference is significant at $p \leq 0.05$

The analysis showed a statistically significant difference between the experimental and control groups at mid-test and post-test. Consequently, the hypothesis that did not expect any significant difference in the mean stress levels between the two groups at pre-test, mid-test and post-test was not accepted. The study concluded that the treatment had a significant effect on stress level at mid-test and post-test based on the pre-test findings.

\section{DISCUSSION}

These results illustrated the beneficial effects of relaxation exercises coupled with the use of sleep masks at night to counteract stress levels in the elderly for a period of eight weeks. These findings are in agreement with studies done either on PMR or the influence of light at night by Cho et al., (2013), Leblanc et al., (2015a, 2015b), Ramakrishnan and Chandran (2013) and Shah et al., (2010). They found that relaxation exercise and/or removal of light at night improved the mental, physical and physiological domains of the elderly. Relaxation exercise therefore promote stress relief and better 
sleep leading to improved quality of life and functional independence. The present study was unique because it combined both relaxation and the effect of removal of light at night on stress level in the elderly. It established the beneficial impact within four weeks of intervention.

The presence of high stress levels in the home for the aged at pre-test was in agreement with Olum (2014) who identified that the majority of the elderly in Kenya are faced with a host of problems ranging from economic, health, and social to personal problems. These stressors lead to adverse effects on the stress coping mechanism of the body among vulnerable populations leading to conditions such as atherosclerosis, hypertension, cancers, osteoporosis, insomnia, arthritis, Type 2 diabetes, dementia, diminished sense of self-worth, backaches, headaches and many more (Bruno, 2013; NFK, 2014; Shah et al., 2010; Shinde et al., 2013). Stress is symptomized by worries, hypervigilance, hyperactivity and a state of alertness; elements predisposing to night time awakenings. A chronic stressful state causes disinterest in life as well as fatigue which tend to foster a declined level of physical activity during the day subsequently contribute to sleep disturbances (Leblanc et al., 2015a, 2015b; Sinha \& Jastreboff, 2013; Slavich, 2016).

These high stress levels could be attributed to the fact that the elderly in long term care facilities frequently report experiencing stress (Olum, 2014) even at bedtime with thoughts pestering them due to lack of control, trepidation, fear of the unknown and extreme worries about the future (Aldao et al., 2010; Barlow \& Durand, 2004; Hofman, Schulz, Heering, Muench \& Bufka, 2010). Despite the elderly being observed as capable of maintaining a high level of motivation, their anxiety at bedtime make all their efforts counterproductive encouraging elevated sleep-onset latency (Barlow \& Durand, 2004; Hofman et al., 2010; Leblanc et al., 2015a, 2015b). The persistent stressful situation of the elderly sleeping badly and the resultant fatigue makes them feel that pharmacological solutions are warranted to negate their existing sleeping problems hence discouraging more proactive measures of managing the said problems (Hofman et al., 2010; Leblanc et al., 2015a, 2015b). These factors were also observed in a home for the elderly in Nairobi and may have been the reason why some participants were on medication.

Research suggests that nonpharmacological interventions such as PMR exercise and sleep masks were equally effective and without side effects as compared to pharmacological solutions (Garber et al., 2011; Yang, 2012). In the present study, the intervention involving relaxation exercise and the removal of light at night may have assisted in the management of this very high stress status. The significant difference demonstrated between the experimental and control groups after four and eight weeks of intervention were important in enhancing the efficacy of these nonpharmacological means of stress management among the elderly. The role of PMR exercises and sleep environment modification in the deterrence of the mentioned health implications resulting from very high stress levels is widely recognized (Garber et al., 2011). These findings support those by Bonnet and Arand, (2016), Garber et al., (2011), Gerber, Lindwall, Lindgård, Börjesson and Jonsdottir, (2013b), Shinde et al., (2013) and Sigfusdottir et al., (2011) who proposed that relaxation buffers the damaging effects of stress because seniors who engage in PMR exercises display fewer stress symptoms such as mood impairment, depression and anxiety.

The PMR exercises and sleep environment enhancement may have contributed to the noticeable reduction in the stress levels of the participants in the experimental group. Research suggests that high unmanaged stress contributes to excessive wear and tear of the body, leading to profound and detrimental repercussions to an elderly's physical and mental health (Sinha \&Jastreboff, 2013; Slavich, 2016; Thoits, 2010). High stress is undoubtedly linked to pathogenesis of arterial heart disease (Blumenthal et al., 2010; Bruno, 2013), adjustment in immune, nervous, muscular and skeletal systems and a decline in physical functioning with time (Kjellberg \& Wadman, 2007; Lambert, G., Schlaich, Lambert, E., Dawood \&Esler, 2010; Segerstrom \& Miller, 2004).

The efficacy of PMR in stress management is further echoed by Muriungi and Ndetei (2013) who stated that one of the most effective types of relaxation techniques is the Progressive Muscle Relaxation (PMR) exercises, oriented toward managing distress associated with stress in geriatric population. Rueggeberg et al., (2012) explained that deleterious physical health is observed in those old people who are both sedentary and have high perceived stress. Bonnet and Arand (2016), state that there is evidence on the efficacy of relaxation and sleep hygiene education in the management of 
sleep disturbances. The PMR exercises are superior over other techniques in treating sleep difficulties as it is a method that the elderly learn fast and can even engage in before falling asleep as well as during longer wake time at night (Shinde et al., 2013; Sinha \& Jastreboff, 2013; Slavich, 2016; Unbehaun et al., 2010).

\section{CONCLUSION}

This investigation concluded that Progressive Muscle Relaxation exercises and the use of sleep masks when sleeping was effective in lowering stress levels of the elderly in the home for the aged. The prevalence of high stress, high blood pressure and poor sleep quality at pre-test in this study concurs with other worldwide studies that have established that these ill health conditions are a true picture of the elderly, particularly in those residing in homes for the aged. The lifestyle modification in the elderly living in the home under study involving PMR exercises and habitual use of sleep masks were established as helpful in the management of distress. This is because these interventions and practices serve as educative channels that add onto the old adult's knowledge, skills and motivation paramount to mitigation of the detrimental effects of stress.

It is widely established that the environment contributes significantly to sleep problems especially because the elderly are disturbed by LAN that hinders melatonin secretion. Inadequate sleep at night which is very common in the elderly due to poor sleep environment becomes a stressor that can lead to stress related health conditions. The development of consistent and satisfying relaxation practices and avoidance of light at night, promotes rest and relaxation which, in turn moderates systolic and diastolic blood pressure. This could either decrease the amount of or need for drug therapy in the elderly.

Similarly, relaxation exercises boost relaxation response which assists the body in confronting stress and hence, curtail mood disorders such as depression which are common in the elderly. Research indicates that, this intervention generates calmness in the body's muscles, not only during the day, but also when the elderly are resting at night, inducing restful sleep. These relaxation exercises appeared to promote a more regular sleep pattern and stress relief in the elderly. These non-pharmacological interventions have been recognized in the current study as being effective in the management of high stress levels in the elderly.

\section{REFERENCES}

[1] Olum, G. (2014). Report on Status and Implementation of National Policy on Ageing in Kenya. United Nations Department of Economic and Social Affairs (UNDESA).www.un.org/esa/socdev/kenya. Retrieved on August 23, 2014.

[2] Bruno, P.W. (2013). Hypertension; Prevention and Management. GRECAH: Nairobi, Kenya.

[3] Harvard Health Publications (2009). Relaxation, Stress and Sleep.www.dartmouth.edu. Accessed on February 14, 2016.

[4] Benson, H., Casey, A. \& Dadoly, A. (eds.). (2008). Stress Management; Approaches for preventing and reducing stress.A Harvard Medical School Special Health Report. Boston, MA: Harvard Medical School.

[5] National Centre for Complementary and Integrative Health. (2016). Relaxation Techniques for Health.www.nccih.nih.gov Accessed on July 24, 2016.

[6] Barnes, P.M., Powell- Griner, E., McFann, K. and Nahin, R.L. (2004). Complementary and Alternative Medicine Use among Adults; United States, 2002. Advance Data from Vital and Health Statistics. No. 343. National Center for Health Statistics.Retrieved May 16, 2014.

[7] Muriungi, S. and Ndetei, D. (2013). Effectiveness of Psycho-education on Depression, Hopelessness, Suicidality, Anxiety and Substance Use among Basic Diploma Students at Kenya Medical Training College. South Africa Journal of Psychology, 2, 41-50.

[8] Shinde, N., Shinde, K. J., Khatri, S.M., Hande, D. \& Bhushan, V. (2013). Immediate Effects of Jacobson's Progressive Muscular Relaxation in Hypertension. Scholars Journal of Applied Medical Sciences, 1, 8085.

[9] Fulghum, D. (2007). Melatonin; New Research Shows Impressive Health Benefits. www.pdrhealth.com Accessed March 16, 2014.

[10] Wilson, H. (2016). The Power of Darkness. Guardian News.www.theguardian.com Accessed on August 14, 2016.

[11] Drake, M., Mills, I. W. and Noble, J. (2004).Melatonin Pharmacotherapy for Nocturia in Men with Benign 
Effects of Relaxation Exercise and Sleep Environment Modification on Stress among Institutionalized Elderly in Nairobi City, Kenya

Prostatic Enlargement. Journal of Urology, 3, 119-202.

[12] Bonnet, M. H. \& Arand, D.L. (2016).Treatment of Insomnia. Sleep Med Rev, 7, 297-310.

[13] Healthwise Staff.(2015). Stress Management; Doing Progressive Muscle Relaxation Exercise.www. uofm health.org. Accessed on July 10, 2016.

[14] The Alternative Daily. (2016). The Importance of Darkness to your Health. www.thealternativedaily.com Accessed on July 20, 2016.

[15] Figueiro, M. G. (2015). Light Therapy for Better Sleep. Sleep Review Journal, 46, 506-519.

[16] Obayashi, K., Saeki, K., Iwamoto, J., Ikada, Y. \& Kurumatani, N. (2013). Exposure to Light At Night and Risk of Depression in the Elderly. Journal of affective disorders, 1, 331-336.

[17] Engle-Friedman, M. (2014).The Effects of Sleep Loss on Capacity and Effort. Sleep Science, 4, 213-224.

[18] Stevens, R. G. (2015). A Dark Night is Good for your Health. www.theconversation.com Accessed on February 24, 2016.

[19] Agency for Healthcare Research and Quality (AHRQ). (2005). In Informed Health Online.(2016). Relaxation Techniques and Sleep Hygiene for Insomnia.www.ncbi.nlm.nih.gov Accessed on March 22, 2016.

[20] Yang, P.Y., Ho, K. H., Chen, H.C. \& Chien, M. Y. (2012).In Informed Health Online.(2016). Relaxation Techniques and Sleep Hygiene for Insomnia.www.ncbi.nlm.nih.gov Accessed on March 22, 2016.

Citation: H. Bulinda, et.al. "Effects of Relaxation Exercise and Sleep Environment Modification on Stress among Institutionalized Elderly in Nairobi City, Kenya" International Journal of Sports and Physical Education (IJSPE), vol 5, no. 4, 2019, pp. 5-11. doi: http://dx.doi.org/10.20431/2454-6380.0504002.

Copyright: () 2019 Authors. This is an open-access article distributed under the terms of the Creative Commons Attribution License, which permits unrestricted use, distribution, and reproduction in any medium, provided the original author and source are credited. 